\title{
Ensemble using different Planetary Boundary Layer schemes in WRF model for wind speed and direction prediction over Apulia region
}

\author{
Andrea Tateo $^{1,2}$, Mario Marcello Miglietta ${ }^{4}$, Francesca Fedele ${ }^{2}$, Micaela Menegotto ${ }^{2}$, Alfonso Monaco $^{3}$, \\ and Roberto Bellotti ${ }^{1,3}$ \\ ${ }^{1}$ Dipartimento Interateneo di Fisica, Università deli Studi di Bari “A. Moro”, Via G. Amendola 173, 70126, \\ Bari, Italy \\ ${ }^{2}$ Apulia Region Environmental Protection Agency (ARPA Puglia), C.so Trieste 27, 70126, Bari, Itali \\ ${ }^{3}$ Istituto Nazionale di Fisica Nucleare (INFN), Sezione di Bari, Via Orabona 4, 70125 Bari, Italy \\ ${ }^{4}$ National Research Council of Italy (CNR), Institute of Atmospheric Sciences and Climate (ISAC), Lecce, Italy \\ Correspondence to: Mario Marcello Miglietta (m.miglietta@isac.cnr.it)
}

Received: 13 January 2017 - Revised: 5 April 2017 - Accepted: 6 April 2017 - Published: 28 April 2017

Abstract. The Weather Research and Forecasting mesoscale model (WRF) was used to simulate hourly $10 \mathrm{~m}$ wind speed and direction over the city of Taranto, Apulia region (south-eastern Italy). This area is characterized by a large industrial complex including the largest European steel plant and is subject to a Regional Air Quality Recovery Plan. This plan constrains industries in the area to reduce by $10 \%$ the mean daily emissions by diffuse and point sources during specific meteorological conditions named wind days. According to the Recovery Plan, the Regional Environmental Agency ARPA-PUGLIA is responsible for forecasting these specific meteorological conditions with $72 \mathrm{~h}$ in advance and possibly issue the early warning.

In particular, an accurate wind simulation is required. Unfortunately, numerical weather prediction models suffer from errors, especially for what concerns near-surface fields. These errors depend primarily on uncertainties in the initial and boundary conditions provided by global models and secondly on the model formulation, in particular the physical parametrizations used to represent processes such as turbulence, radiation exchange, cumulus and microphysics. In our work, we tried to compensate for the latter limitation by using different Planetary Boundary Layer (PBL) parameterization schemes. Five combinations of PBL and Surface Layer (SL) schemes were considered.

Simulations are implemented in a real-time configuration since our intention is to analyze the same configuration implemented by ARPA-PUGLIA for operational runs; the validation is focused over a time range extending from 49 to $72 \mathrm{~h}$ with hourly time resolution. The assessment of the performance was computed by comparing the WRF model output with ground data measured at a weather monitoring station in Taranto, near the steel plant. After the analysis of the simulations performed with different PBL schemes, both simple (e.g. average) and more complex post-processing methods (e.g. weighted average, linear and nonlinear regression, and artificial neural network) are adopted to improve the performances with respect to the output of each single setup. The neural network approach comes out as the most promising method. 


\section{Introduction}

Due to the numerous and high-impact industrial activities, Taranto (south-eastern Italy) is included in the so-called areas at "high risk of environmental crisis". In fact, it hosts a heavy industrial district, unfavourably positioned very close to residential areas. Among these industrial plants, particular attention is addressed to the steelworks: the ILVA plant is extended over a surface of 15 million square meters, including $200 \mathrm{~km}$ of railway tracks, $50 \mathrm{~km}$ of roads, $190 \mathrm{~km}$ of conveyor belts, 5 blast furnaces and 5 converters. Storage and handling of primary materials in the stockyards and transportation of materials by heavy duty trucks are the major sources of particulate. The most critical neighbourhood, called Tamburi, is located at less than $1 \mathrm{~km}$ from the stockyard, downwind of the plant with respect to the prevailing north-westerly wind.

In the last few years, particles under $10 \mu \mathrm{m}\left(\mathrm{PM}_{10}\right)$ and benzo(a)pyrene $(\mathrm{B}(\mathrm{a}) \mathrm{P})$ concentrations measured in Tamburi exceeded the limit permitted by law several times (Trizio et al., 2016). A study of these critical pollution events showed a close correlation with wind conditions, in particular the exceedances occur when the wind favours the pollutant transport from the industrial site to the adjacent urban area (Amodio et al., 2013). Based on the statistical analysis of meteorological measurements taken in a monitoring site near the coastline during several years, a criterion for the daily identification of these events named "wind days" was defined: these critical days are characterized by at least 3 consecutive hours of wind coming from $\mathrm{N}-\mathrm{NW}$ and speed higher than $7 \mathrm{~m} \mathrm{~s}^{-1}$. This criterion is part of a Regional Air Quality Recovery Plan, implemented to improve the air quality in the Tamburi district. This plan determines that, in case of prediction of wind days, the alert should be sent two days in advance so that industrial plants must reduce the mean daily emissions by diffuse and point sources by $10 \%$. According to this plan, the Regional Environmental Agency ARPAPUGLIA runs the WRF model to forecast wind speed and direction every day up to hour 72 , considering the forecast range from 49 to $72 \mathrm{~h}$ for issuing alerts.

For the above reasons, to predict well in advance the occurrence of "wind days", it is necessary to accurately simulate wind speed and direction. In this effort, the Weather Research and Forecasting model (WRF) was used to simulate hourly $10 \mathrm{~m}$ wind speed and direction over the city of Taranto (Fedele et al., 2014, 2015). WRF is a consolidate mesoscale numerical weather prediction system, which represents the state-of-the-art in meteorological limited area modelling. This model has been successfully used in the past to simulate the evolution of boundary layer features over Apulia region (e.g., De Tomasi et al., 2011; Comin et al., 2015). Unfortunately, meteorological model outputs, particularly near-surface fields (e.g., precipitation, wind, ...), may be affected by significant errors. These errors depend primarily on the uncertainties of the initial and boundary condi- tions provided by global models and secondly on the physical parametrizations used to represent processes such as turbulence, radiation exchange, cumulus and microphysics. Thus, an optimized method or postprocessing procedure to reduce the simulation errors is very important in order to improve the model results (Tateo et al., 2015). Many papers demonstrate that an ensemble of model runs, generated for example by using different PBL parameterization schemes, may improve the predictive skill compared to a single deterministic run (Evans et al., 2012).

In the present work, we considered a total of five combinations of PBL and Surface Layer (SL) schemes, i.e. the Yonsei University with topographic correction, Mellor-YamadaJanjic and Mellor-Yamada-Nakanishi-and-Niino level 2.5 schemes for PBL parametrization; for the latter, three compatible SL schemes are implemented to analyse the specific effect of SL schemes. Simulations are considered in real-time implementation: the validation is performed for a forecast range from 49 to $72 \mathrm{~h}$ with hourly time resolution. We compared the results from each single model setup with different linear and nonlinear post-processing approaches as described in Sect. 2. Section 3 deals with the results of wind speed and direction analysis. In Sect. 4, the discussion and interpretation of the results are shown.

\section{Material and method}

Hourly $10 \mathrm{~m}$ wind speed and direction in Taranto are obtained by running the WRF model using five different combinations of PBL (and associated SL) schemes as reported in Table 1. In subsequent sections, we will refer to the " $i$ " run to indicate the " $i$ "th combination of PBL and SL schemes as reported in Table 1.

In each run, all other parameterization schemes were kept unchanged. We considered two nesting domains with 16 and $4 \mathrm{~km}$ grid spacing respectively and 40 eta levels. The model configuration can be summarized as follows: the "Thompson scheme" microphysics, for longwave and shortwave radiation the "rrtm scheme" and "Dudhia scheme" respectively, the effect of resolved and unresolved orography on surface wind as in Jiménez and Dudhia (2012), the Kain-Fritsch cumulus parameterization and the Noah land-surface model.

The analysis was performed over a period of eight months, from 1 August 2015 to 31 March 2016. Simulations are implemented in a real-time configuration since our intention is to analyze the results for the operational runs implemented at ARPA-PUGLIA; for validation purposes, the range from 49 to $72 \mathrm{~h}$ with hourly resolution is considered. The Global Forecast System (GFS) analyses/forecasts were used as initial/boundary conditions.

To compare the WRF outputs with the weather monitoring station measurements, we used the nearest neighbor approach to the model fields interpolated by Grid Analysis and Display System (GrADS). 
Table 1. Combination of Boundary Layer and Surface Layer Schemas considered in the present work.

\begin{tabular}{|c|c|c|}
\hline Model & Boundary Layer Schemes & Surface Layer Schemes \\
\hline 1 & $\begin{array}{l}\text { Yonsei University with topographic correction } \\
\text { (YSU) }\end{array}$ & $\begin{array}{l}\text { Revised MM5 Monin-Obukhov } \\
\text { (Jimenez, renamed in v3.6) }\end{array}$ \\
\hline 2 & Mellor-Yamada-Janjic (Eta) TKE & Monin-Obukhov (Janjic Eta) \\
\hline 3 & $\begin{array}{l}\text { Mellor-Yamada Nakanishi and Niino level } 2.5 \\
\text { (MYNN } 2.5 \text { level TKE) }\end{array}$ & $\begin{array}{l}\text { Revised MM5 Monin-Obukhov } \\
\text { (Jimenez, renamed in v3.6) }\end{array}$ \\
\hline 4 & $\begin{array}{l}\text { Mellor-Yamada Nakanishi and Niino level } 2.5 \\
\text { (MYNN } 2.5 \text { level TKE) }\end{array}$ & Monin-Obukhov (Janjic Eta) \\
\hline 5 & $\begin{array}{l}\text { Mellor-Yamada Nakanishi and Niino level } 2.5 \\
\text { (MYNN } 2.5 \text { level TKE) }\end{array}$ & Mellor-Yamada Nakanishi and Niino (MYNN) \\
\hline
\end{tabular}

\subsection{Wind speed}

The wind speed prediction errors ES (bias) were obtained as difference between the WRF model outputs $\mathrm{WS}_{\text {pred }}$ and the measured data at the weather monitoring station $\mathrm{WS}_{\mathrm{obs}}$ according to the Eq. (1):

$\mathrm{ES}=\mathrm{WS}_{\text {pred }}-\mathrm{WS}_{\mathrm{obs}}$

The WS correction performances were estimated by considering the mean hourly error over the full dataset. After obtaining five different wind speed predictions by running each model configuration in Table 1, six different post-processing techniques were considered to obtain a more accurate wind speed prediction $\left(\mathrm{WS}_{\text {best }}\right)$ : simple average, weighted average (we estimated the weight by considering the prediction errors in a window of 30 days), linear regression, Kalman Filter method combined with an artificial neural network in the stacked generalization procedure, two artificial neural networks combined in the stacked generalization procedure, and an individual artificial neural network. In following subsections, a brief explanation is given for the last three, more complex, methods.

\subsubsection{Kalman Filter method combined with artificial neural network in the stacked generalization procedure}

The Kalman Filter (KF) method is a recursive algorithm commonly used to estimate an unknown variable at time " $t$ ", using a series of observations of that variable, measured in a previous period. This method is based on the Bayesian inference and allows considering statistical noise and other inaccuracies (Evensen, 2003).

In our analysis, we used the Kalman Filter to evaluate, for each model and for each daily hour, the error at time " $t$ ", by measuring the errors in the previous week. We suppose the error remains constant in this time window, except for the statistical noise $\alpha$ as shown in Eq. (2):

$\mathrm{ES}_{i}(t, h)=\mathrm{ES}_{i}(t-1, h)+\alpha(h)$
In this way, the prediction of each model is improved. To combine the five corrected predictions, we used a simple artificial neural network (ANN) in the stacked generalization method (Wolpert, 1992).

The best architecture in terms of minimum Root Mean Square Error (RMSE) is found to be a feed-forward multilayer perceptron, one hidden layer with three neurons, and seven input neurons, five for the corrected predictions and two for the hourly cyclical component, $H_{1}$ and $H_{2}$, represented in Eqs. (3) and (4) as:

$H_{1}=\sin \left(\frac{h \cdot \pi}{24}\right)$

$H_{2}=\cos \left(\frac{h \cdot \pi}{24}\right)$

The best size in terms of Root Mean Square Error of the training period is found to be 20 days. Summarizing, we need a period of 27 days to estimate $\mathrm{WS}_{\text {best }}$, the first seven of which are used to initialize the KF correction.

\subsubsection{Two artificial neural networks combined in the stacked generalization procedure}

This procedure is similar to the procedure described in the Sect. 2.1.1. The KF algorithm is replaced with an additional ANN. In order to correct each model configuration, a dedicated ANN is necessary. The best architecture in terms of RMSE is found to be a feed-forward multilayer perceptron, one hidden layer with two neurons, and three input neurons, one for the predicted wind speed provided by each single model and two for the hourly cyclical component. To train this ANN, the best size period in terms of RMSE is found to be 10 days.

Also in this procedure, to combine the five corrected values, we used an additional ANN in the Stacked Generalization method, similar to that described in Sect. 2.1.1. For the additional ANN, the best size of the training period in terms 


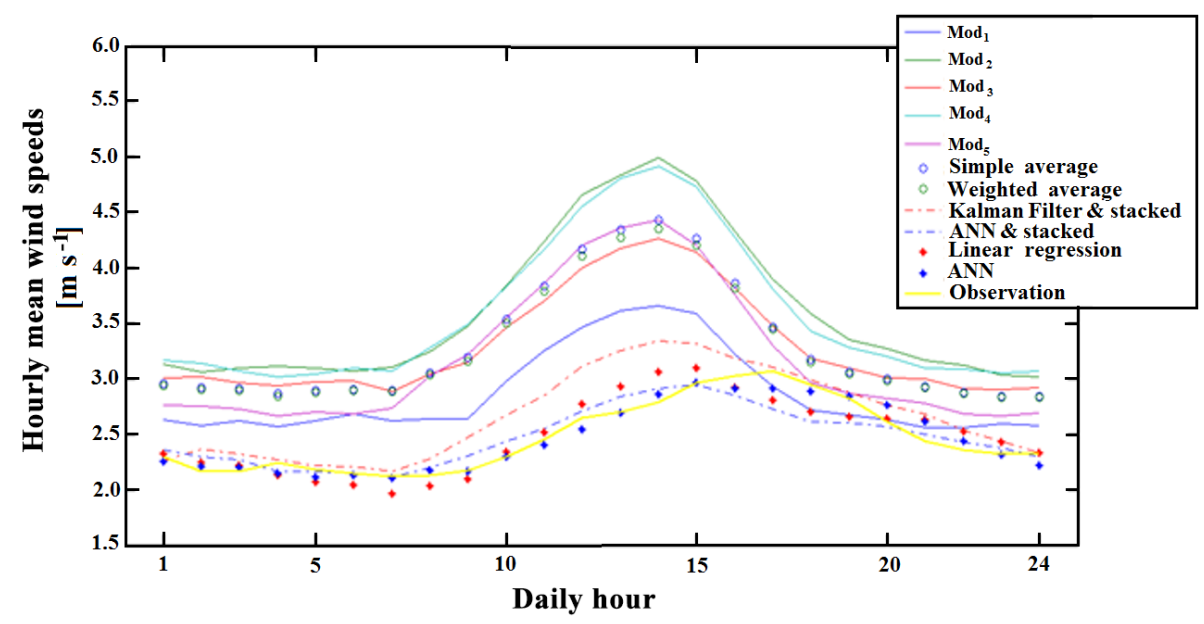

Figure 1. Comparison of hourly mean values of wind speed using the five selected model configurations and their post-processed value with respect to the observed data.

of RMSE is found to be 20 days. Summarizing, we need a period of 30 days to estimate $\mathrm{WS}_{\text {best }}$, the first ten of which are used to train the five ANNs needed to correct, separately, each single model.

\subsubsection{Individual artificial neural network}

The ANN is a nonlinear regression approach (Gardner and Dorling, 1998). To evaluate the best architecture, i.e. to estimate the best size of the training period and the best input features, various tests are implemented. The best configuration in terms of RMSE is found to be a feed-forward multilayer perceptron architecture, one hidden layer with three neurons, and seven input neurons, five for the corrected predictions and two for the hourly cyclical component, $H_{1}$ and $\mathrm{H}_{2}$, as represented in Eqs. (3) and (4). The best size of the training period in terms of RMSE is found to be 30 days. In this approach, the task of the individual ANN is to correct each model and combine them simultaneously.

\subsection{Wind direction}

Throughout this study, the wind direction prediction error ED was obtained by comparing the WRF outputs $\mathrm{WD}_{\text {pred }}$ with the measured data $\mathrm{WD}_{\mathrm{obs}}$ according to Eq. (5):

$\mathrm{ED}=$

$\left\{\begin{array}{l}\mathrm{WD}_{\text {pred }}-\mathrm{WD}_{\text {obs }} \\ -|360-| \mathrm{WD}_{\text {pred }}-\mathrm{WD}_{\mathrm{obs}} \\ +|360-| \mathrm{WD}_{\text {pred }}-\mathrm{WD}_{\mathrm{obs}}\end{array} \mid\right.$

if $\left|\mathrm{WD}_{\text {pred }}-\mathrm{WD}_{\text {obs }}\right| \leq 180^{\circ}$

if $\left(W_{\text {pred }}-W_{\text {obs }}\right)>180^{\circ}$

if $\left(\mathrm{WD}_{\text {pred }}-\mathrm{WD}_{\mathrm{obs}}\right)<-180^{\circ}$

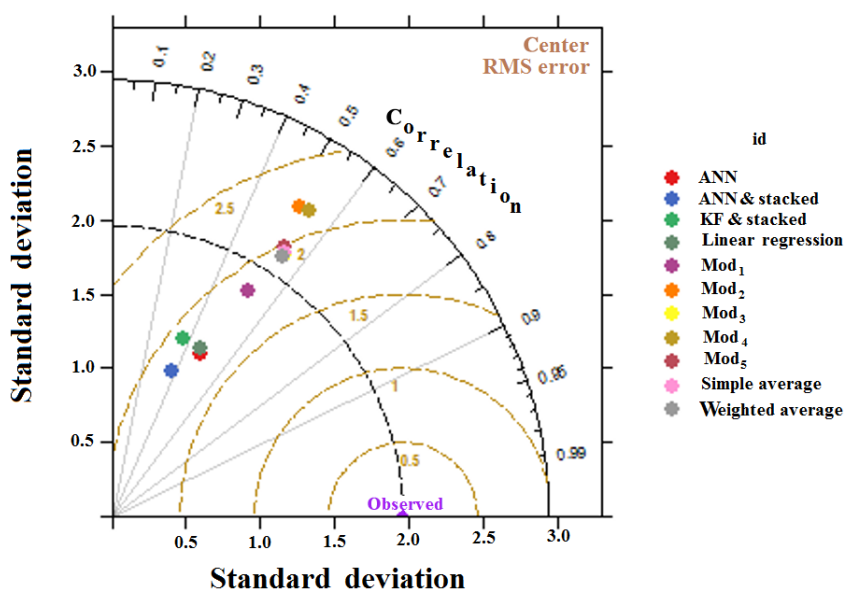

Figure 2. Taylor Diagram to compare the five models and their combinations with respect to the observed data in terms of Centered Root Mean Square Error, Correlation Coefficient, and Standard Deviation.

The performances are given in terms of Direction Accuracy (DACC) expressed by Eq. (6):

DACC $($ Angle_ref $)=$

number of cases with error lower than Angle_ref number of total cases

that represents the percentage of errors (in absolute value) lower than a reference value (Angle_ref).

First, a partial average $\overline{\mathrm{WD}_{\mathrm{par}}}$ is estimated considering only the model outputs within a standard deviation from the total average. Next, the $\overline{\mathrm{WD}_{\mathrm{par}}}$ estimate is corrected by a linear regression trained in time: thus, the error $\mathrm{ED}_{\text {best }}$ is obtained as:

$\mathrm{ED}_{\text {best }}=A_{1} \cdot \overline{\mathrm{WD}_{\text {par }}}+A_{2}$, 


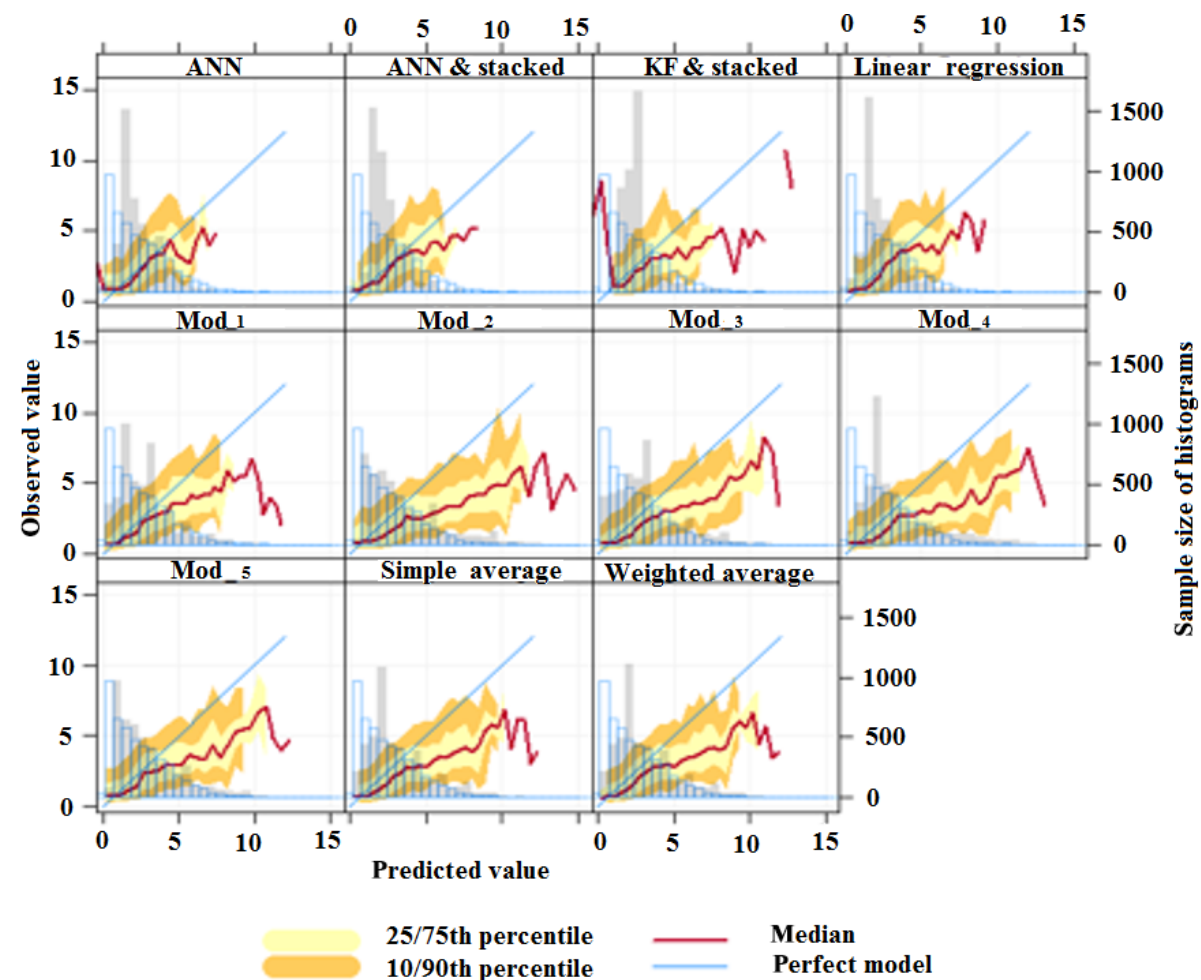

Figure 3. Conditional Quantile Plot to compare the five model configurations and their post-processed combinations with respect to the observed data in terms of distribution.

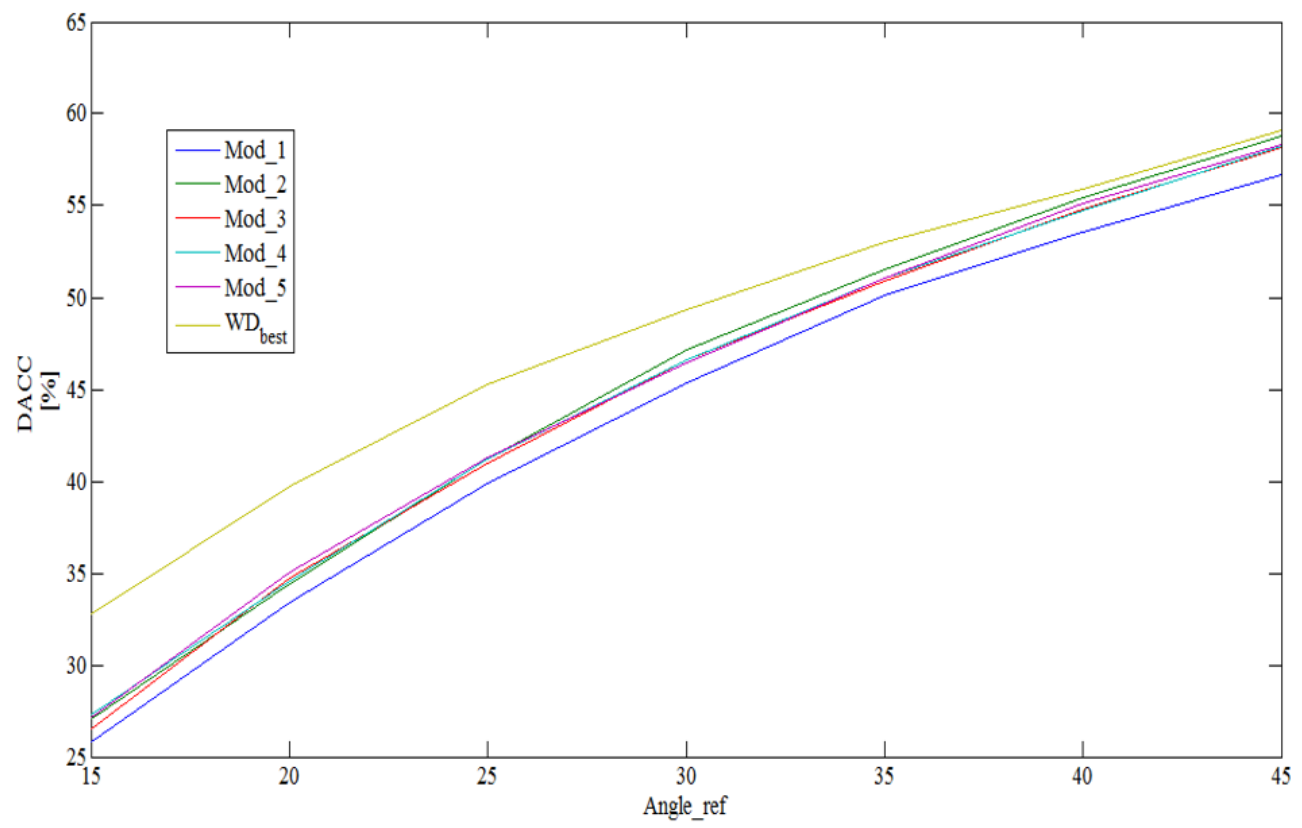

Figure 4. Direction Accuracy plot versus Angle_ref (see text for definition) for each model and correction. Only values smaller than $45^{\circ}$ are shown because no improvement is found for larger values. 


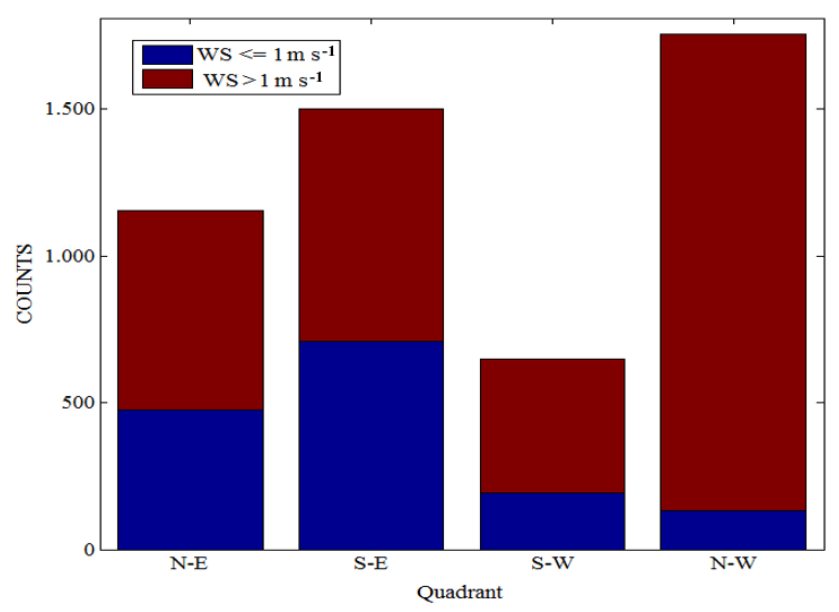

Figure 5. Bar Diagram of data subdivided by quadrant. For each quadrant, the frequencies are reported below and above the threshold of $1 \mathrm{~m} \mathrm{~s}^{-1}$.

where the coefficients $A_{1}$ and $A_{2}$ are estimated by training over the selected time window. Consequently, the correct $\mathrm{WD}_{\text {best }}$ is estimated by Eq. (8)

$\mathrm{WD}_{\text {best }}=\overline{\mathrm{WD}_{\text {par }}}+\mathrm{ED}_{\text {best }}$.

The analysis was carried out separately for each quadrant (N-E, S-E, S-W, N-W) because WD errors are found to be quadrant-dependent. Although each model presents a WD error distribution centered at zero when the full domain [0$360^{\circ}$ ] is considered, when each quadrant is considered separately the WD error distributions are found to be not centered at zero.

\section{Results}

\subsection{Wind speed results}

The analysis shows that all considered model configurations present a positive mean bias. In fact, Fig. 1 shows that all model runs (colour continuous lines) simulate a wind speed that is on average larger than the observed values (yellow continuous lines). For this reason, the simple average (blue small circles) and the weighted average (green small circles) cannot improve the performances. Therefore, as described in Sect. 2, we implemented four post-processing methods based on the training with past data. The Artificial Neural Network (blue stars) is found to be the best procedure to combine the five wind speed predictions, reproducing the mean hourly wind speed very well. Similarly, the Taylor diagram in Fig. 2 shows that ANN is better also in terms of centered RMSE and correlation. Conditional Quintile Plots are reported in Fig. 3. The conditional quantile plot splits the predicted values into evenly spaced bins and calculates the median, the 25/75th percentiles, and the 10/90th percentiles; also, for each bin the corresponding values of the observations are identified

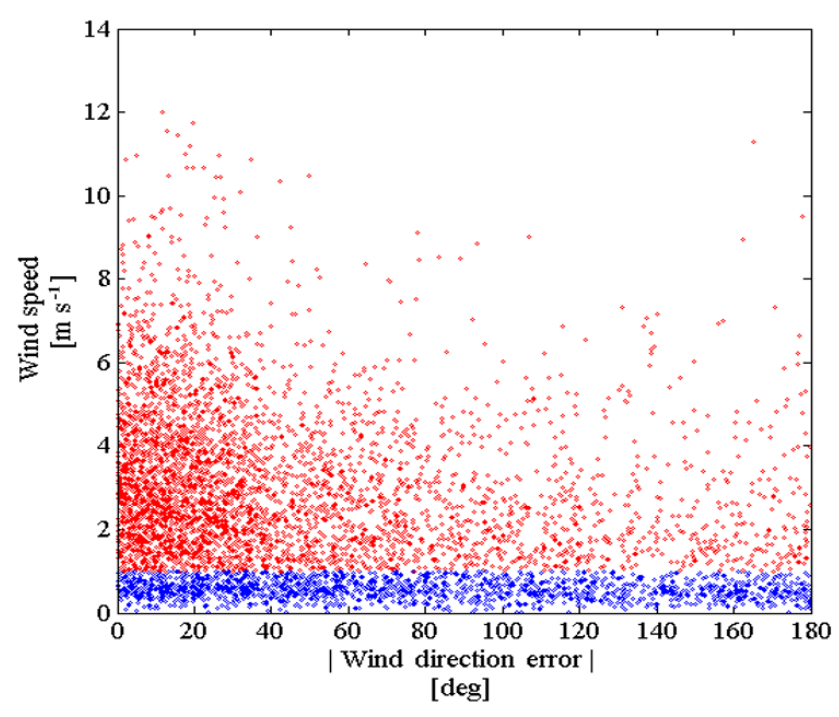

Figure 6. Scatter Diagram between wind speeds and wind direction errors. (WS values smaller than $1 \mathrm{~m} \mathrm{~s}^{-1}$ are shown in blue, the other ones in red)

(Wilks, 2005). Additionally, in each subplot, the observed WS distribution (histogram with blue-contoured columns), and the predicted WS distribution (grey histogram) are reported. A good performance is obtained when the median (red line) coincides with the bisector (blue straight line) and when the spread in the percentile is as small as possible.

Some remarks can be deduced from Fig. 3:

- On average, the five original WRF model configurations show values higher than observed, i.e. the medians are under the bisectors;

- Although model 5 shows a WS distribution very similar to the observed distribution, it shows a great error in terms of median due to data mismatching;

- The ANN shows a better mean WS correction: effectively, its median overlaps the bisector well at least for WS smaller than $5 \mathrm{~ms}^{-1}$; the worse performance for higher values is due to their limited presence in the training dataset, as shown in the histograms of Fig. 3: a limited presence implies a worse training of ANN for these high values.

\subsection{Wind direction results}

The analysis shows that all models have wide WD error distributions. In contrast with the WS analysis, the WD analysis does not include the parameter "daily hour" because the WD error is found to be independent of it. The application of the procedure described in Sect. 2.2 shows an improvement in terms of DACC for reference values smaller than $45^{\circ}$, while no improvement is found for larger discrepancies, which are anyway less present in the dataset (Fig. 4). 


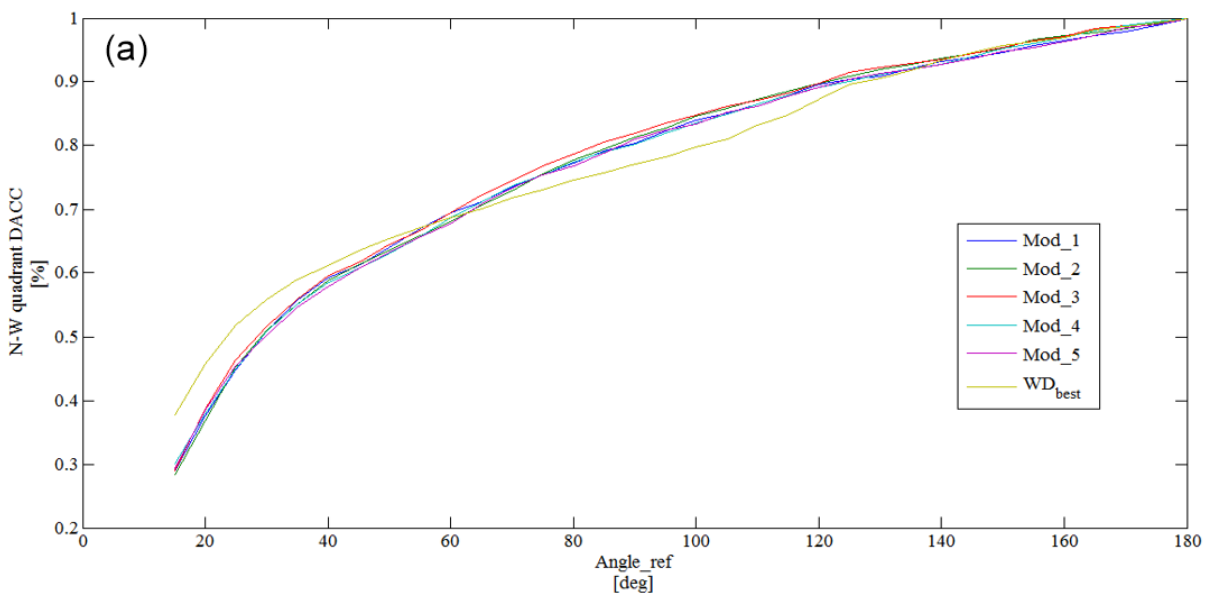

\section{FULL}

DATASET

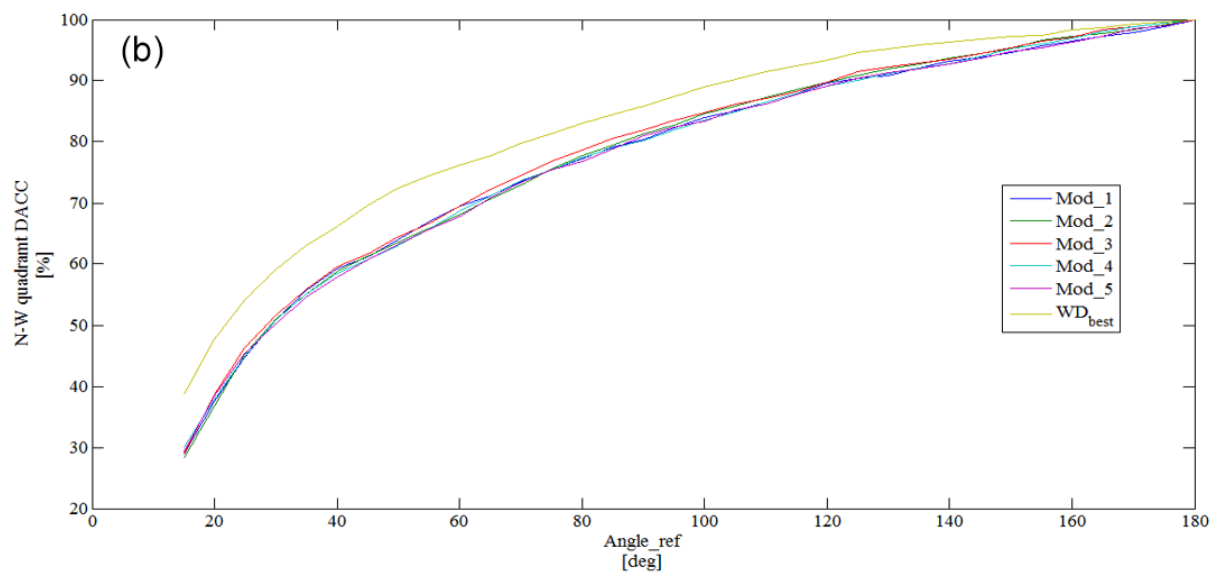

\section{CASES WITH}

WS $>1 \mathrm{~m} \mathrm{~s}^{-1}$

Figure 7. (a) Direction Accuracy plot versus all possible Angle_ref for each model and correction using the full dataset related to N-W quadrant. (b) Direction Accuracy plot versus all possible Angle_ref for each model and correction using only data with wind speed greater than $1 \mathrm{~m} \mathrm{~s}^{-1}$ related to $\mathrm{N}-\mathrm{W}$ quadrant.

The quadrant analysis shows that the improvement is greater for the Nord-West quadrant (not shown) and we focus on this subset of directions in the following. This improvement is due to the higher frequency of occurrence of wind direction from this quadrant (better trained network), and for its higher intensity (Fig. 5). In fact, higher wind speeds imply smaller WD error: Fig. 6 shows that WS values smaller than $1 \mathrm{~m} \mathrm{~s}^{-1}$ are associated with WD errors uniformly distributed between 0 and $180^{\circ}$, while for higher WS the points cluster in the area of smaller WD errors (the Kolmogorov-Smirnov test indicates a dependence on WS with a $p$-value $<0.05$ ). Since the interest of present work is on wind day, we chose to exclude from the following analysis all cases characterized by observed WS smaller than $1 \mathrm{~m} \mathrm{~s}^{-1}$. Figure 7 shows that using, this additional constraint, the correction in the $\mathrm{N}-\mathrm{W}$ quadrant is further improved, even for higher values of WD, which generally suffered from a limited benefit in the use of the ANN. However, the improvement for the other quadrants is less effective, due to the lower frequency of cases with WS greater than $1 \mathrm{~m} \mathrm{~s}^{-1}$ (Fig. 6).

\section{Conclusions}

With the purpose of studying and improving $10 \mathrm{~m}$ wind speed and direction simulations over Taranto, a city in southeastern Italy including the largest European steel plant, the WRF model was employed with a total of five different combinations of PBL and SL schemes. Simulations are considered in real-time implementation, and are evaluated over a forecast range extending from 49 to $72 \mathrm{~h}$ with hourly time resolution over a period of eight months. The analysis shows that all considered model configurations present a positive mean bias for the wind speed. Instead, for the wind direction, the analysis shows that all considered model configurations present a wide error distribution centered at zero.

To improve the wind speed predictions, six different methods are considered. The analysis shows that the simple average and weighted average are not able to improve the performances. Among the other four methods based on training with past data, the ANN is found able to better eliminate the mean bias, to better reduce the centered RMSE, and shows 
a better overlap between the median values and the observations.

To improve the wind direction predictions, since neural networks were not effective in improving the results, a dedicated procedure was implemented, based on the partial average and on the linear regression. The proposed method is found to improve the wind direction prediction error of each model in terms of direction accuracy. A better improvement is found when the analysis is carried out separately for each quadrant, and by excluding the data with WS less than $1 \mathrm{~m} \mathrm{~s}^{-1}$. In particular, a better result is found for the $\mathrm{N}-\mathrm{W}$ quadrant due to his higher frequency of cases from this direction.

Competing interests. The authors declare that they have no conflict of interest.

Edited by: D. Reinert

Reviewed by: two anonymous referees

\section{References}

Amodio, M., Andriani, E., de Gennaro, G., Di Gilio, A., Ielpo, P., Placentino, C. M., and Tutino, M.: How a Steel Plant Affects Air Quality of a Nearby Urban Area: A Study on Metals and PAH concentrations, Aerosol Air Qual. Res., 13, 497-508, 2013.

Comin, A. N., Miglietta, M. M., Rizza, U., Acevedo, O. C., and Degrazia, G. A.: Investigation of a sea-breeze convergence in a Mediterranean peninsula, Atmos. Res., 160, 68-79, 2015.

De Tomasi, F., Miglietta, M. M., and Perrone, M. R.: The growth of the PBL in a coastal site: a case study, Boundary Layer Meteorology, 139, 521-541, 2011.

Evans, J. P., Ekström, M., and Ji, F.: Evaluating the performance of a WRF physics ensemble over South-East Australia, Clim. Dynam., 39, 1241-1258, 2012.
Evensen, G.: The ensemble Kalman filter: Theoretical formulation and practical implementation, Ocean Dynam., 53, 343-367, 2003.

Fedele, F., Menegotto, M., Trizio, L., Angiuli, L., Guarnieri Calò Carducci, A., Bellotti, R., Giua, R., and Assennato, G.: Meteorological effects on pm10 concentrations in an urban industrial site: a statistical analysis, in: Conference Proceedings 1st International Conference on Atmospheric DUST, 162-167, doi:10.14644/dust.2014.026, ISSN: 2283-5954, 2014.

Fedele, F., Miglietta, M. M., Perrone, M. R., Burlizzi, P., Bellotti, R., Conte, D., and Guarnieri Calò Carducci, A.: Numerical simulations with the WRF model of water vapour vertical profiles: A comparison with LIDAR and radiosounding measurements, Atmos. Res., 166, 110-119, 2015.

Gardner, M. W. and Dorling, S. R.: Artificial neural networks (the multilayer perceptron) - a review of applications in the atmospheric sciences, Atmos. Environ., 32, 2627-2636, 1998.

Jiménez, P. A. and Dudhia, J.: Improving the representation of resolved and unresolved topographic effects on surface wind in the WRF model, J. Appl. Meteor. Clim., 51, 300-316, 2012.

Tateo, A., Bellotti, R., Fedele, F., Guarnieri Calò Carducci, A., and Pollice, A.: Post-processing of the Weather Research and Forecasting (WRF) mesoscale model by Artificial Neural Networks, GRASPA-SIS Biennial Conference, Bari, ISBN 978-88-8879377-1, 2015.

Trizio, L., Angiuli, L., Menegotto, M., Fedele, F., Giua, R., Mazzone, F., and Assennato, G.: Effect of the Apulia air quality plan on PM10 and benzo (a) pyrene exceedances, Global Journal of Environmental Science and Management, 2, 95-104, 2016.

Wilks, D. S.: Statistical Methods in the Atmospheric Sciences, Volume 91, Second Edition (International Geophysics), 2nd Edition, Academic Press, 2005.

Wolpert, D.: Stacked Generalization, Neural Networks, 5, 241-259, 1992. 